\title{
Cobalt-chromium versus titanium alloy rods for correction of adolescent idiopathic scoliosis based on 1-year follow-up: a multicenter randomized controlled clinical trial
}

\author{
Daisuke Sakai, MD, PhD, ${ }^{1}$ Masato Tanaka, MD, PhD, ${ }^{2}$ Jun Takahashi, MD, PhD, ${ }^{3}$ \\ Yuki Taniguchi, MD, PhD, ${ }^{4}$ Jordy Schol, MSc, ${ }^{1}$ Akihiko Hiyama, MD, PhD, ${ }^{1}$ \\ Haruo Misawa, MD, PhD, ${ }^{5}$ Shugo Kuraishi, MD, PhD, ${ }^{3}$ Hiroki Oba, MD, $\mathrm{PhD},{ }^{3}$ \\ Yoshitaka Matsubayashi, MD, PhD, ${ }^{4}$ So Kato, MD, PhD, ${ }^{4}$ Ryo Sugawara, MD, PhD, ${ }^{6}$ \\ Masato Sato, MD, PhD, ${ }^{1}$ Masahiko Watanabe, MD, PhD, ${ }^{1}$ and Katsushi Takeshita, MD, PhD ${ }^{6}$ \\ 'Department of Orthopaedic Surgery, Surgical Science, Tokai University School of Medicine, Isehara, Kanagawa; ${ }^{2 D e p a r t m e n t}$ \\ of Orthopaedic Surgery, Okayama Rosai Hospital, Okayama; ${ }^{3}$ Department of Orthopaedic Surgery, Shinshu University School \\ of Medicine, Nagano; ' Department of Orthopaedic Surgery, The University of Tokyo; ${ }^{5}$ Department of Orthopaedic Surgery, \\ Okayama University School of Medicine, Okayama; and ${ }^{6}$ Department of Orthopaedics, Jichi Medical University, Tochigi, Japan
}

\begin{abstract}
OBJECTIVE For instrumented correction surgery for adolescent idiopathic scoliosis (AIS), surgeons are increasingly switching from titanium (Ti) alloy rods to stiffer cobalt-chromium $(\mathrm{CoCr})$ rods. The authors conducted the first multicenter randomized controlled clinical trial to investigate whether these materials affect the outcomes in terms of spine correction and quality of life (QOL). This trial was registered at UMIN Clinical Trials Registry on September 3, 2012, under the identifier UMIN000008838 (level of evidence 1).

METHODS Female AIS patients (Lenke types 1-3, patient age 10-19 years) were recruited at 5 Japanese institutions and randomized into two cohorts: 6.0-mm-diameter Ti rods were placed in one group, and 6.0-mm-diameter $\mathrm{CoCr}$ rods were placed in the other. Patients were followed up at 2 weeks and 3, 6, and 12 months with radiographic examination to quantify the sagittal and coronal correction (Cobb angle, thoracic kyphosis, rib hump, and apical vertebral rotation). Patients completed questionnaires (Scoliosis Research Society-22r, 12-Item Short-Form Health Survey, and Scoliosis Japanese Questionnaire-27) at 6 and 12 months to assess QOL.
\end{abstract}

RESULTS A total of 69 AIS patients were randomized to the demographically similar $\operatorname{Ti}(n=37)$ or $\operatorname{CoCr}(n=32)$ cohort. Four adverse events were recorded, two in each cohort, but these were not related to the rod material. At the final followup, both $\mathrm{Ti}$ and $\mathrm{CoCr}$ cohorts showed significant improvement in spinal correction, including the Cobb angle, thoracic kyphosis, and rib hump size. The correction rates were $68.4 \%$ and $67.1 \%$ for the Ti and $\mathrm{CoCr}$ cohorts, respectively. No parameters differed significantly between the cohorts at any time. Survey data showed improved but similar outcomes in both cohorts.

CONCLUSIONS Both treatments ( $\mathrm{Ti}$ and $\mathrm{CoCr}$ ) produced similar results and were efficient in engendering clinically significant spine corrections.

Clinical trial registration no.: UMIN000008838 (UMIN Clinical Trials Registry)

https://thejns.org/doi/abs/10.3171/2020.9.SPINE201486

KEYWORDS scoliosis; deformity; rod; cobalt-chromium; titanium

A DOLESCENT idiopathic scoliosis (AIS) has an estimated prevalence of $0.5 \%-5.2 \%$, exhibiting generally larger deformities and prevalence in females. ${ }^{1}$ Despite its relatively high prevalence, the etiology of AIS remains largely unknown. ${ }^{2}$ The goal of contemporary correctional AIS surgery is to revise the triplanar spinal deformity while preserving spinal balance and, where feasible, the mobility of the spine segments. Surgery is directed

ABBREVIATIONS AIS = adolescent idiopathic scoliosis; $\mathrm{AVR}=$ apical vertebral rotation; $\mathrm{CoCr}=$ cobalt-chromium; $\mathrm{PJK}=$ proximal junctional kyphosis; $\mathrm{QOL}=$ quality of life; SF-12 = 12-Item Short-Form Health Survey; SRS-22r = Scoliosis Research Society-22r; SS = stainless steel; Ti = titanium.

SUBMITTED August 12, 2020. ACCEPTED September 21, 2020.

INCLUDE WHEN CITING Published online March 19, 2021; DOI: 10.3171/2020.9.SPINE201486. 
at preventing neurological risk, alleviating or preventing disability, improving the patient's self-image, and limiting the deformity progression. Modern surgical intervention generally involves the creation of spinal anchor points to enable spinal manipulation via rigid implants, which are then fixed and fused into place. Successful spine corrections rely on a delicate interplay of the forces applied by the implanted rods via a limited number of anchor points onto the spine. To engender appropriate corrections, the rod rigidity must exceed the rigidity of the spine while not eclipsing the screw-bone interaction capacity.

AIS surgery has undergone a rapid evolution, ${ }^{3}$ including the rod materials used. Initially, stainless steel (SS) was the most commonly used alloy, but since the 2000s there has been a shift toward titanium (Ti) alloy, ${ }^{4}$ mainly due to issues with biocompatibility, infection rates, and MRI distortions ${ }^{5-7}$ Currently, a trend toward cobalt-chromium $(\mathrm{CoCr})$ alloys to replace Ti rods is ongoing. $\mathrm{CoCr}$ is a stronger material and has fewer disadvantages than SS, such as better MRI compatibility and biocompatibility. ${ }^{6,7}$

Although, in theory, $\mathrm{CoCr}$ is a promising material, its effectiveness relative to Ti has not been studied thoroughly for AIS surgery. The literature indicates contradictory conclusions based on data derived mainly from retrospective, nonrandomized, single-institution studies. ${ }^{8-11}$ The clinical effects associated with either implant are likely to be highly dependent on the surgeon's preference, familiarity, and skill. Understanding any potential benefits of switching to $\mathrm{CoCr}$ requires the prospective assessment of Ti rod- and CoCr rod-mediated correction surgery performed by multiple surgeons at different medical institutions. Moreover, these studies merely address differences in radiographical outcomes but do not examine differences in quality of life (QOL). Here, we describe a prospective, randomized, multicenter clinical trial aimed to assess differences in coronal and sagittal curvature corrections and improvements in QOL (by multiple questionnaires) between $\mathrm{Ti}$ rod- and $\mathrm{CoCr}$ rod-mediated correctional surgery for female AIS patients. We hypothesized that the stiffer $\mathrm{CoCr}$ rod can generate larger corrections that will consequently lead to improvements in QOL.

\section{Methods}

This study is a prospective, randomized, multicenter, open-label, single-blinded clinical study (trial no. UMIN000008838, UMIN Clinical Trials Registry) designed to compare the effectiveness of $\mathrm{CoCr}$ and $\mathrm{Ti}$ rods in the surgical correction of AIS. Cohort recruitment was performed between July 2012 and October 2016. Before the initiation, all study procedures were approved by each institution's committee on ethics and institutional review boards. All protocols are in accordance with the ethical standards set by the 1964 Helsinki Declaration and its later amendments.

\section{Patient Selection and Randomization}

Sixty-nine patients with AIS from 5 Japanese academic institutions (Tokai University School of Medicine, Isehara; The University of Tokyo Hospital, Tokyo; Okayama University Hospital, Okayama; Shinshu University School of Medicine, Nagano; and Jichi Medical University Hospital, Tochigi) were recruited for this study. The inclusion criteria were 10- to 19-year-old female patients diagnosed with AIS and the ability to understand and consent to participate in this study. Further inclusion criteria involved having Lenke type 1-3 scoliosis with a Cobb angle of $45^{\circ}$ or more. The exclusion criteria included bone metabolism complications; infectious disease; neuromuscular disease; having undergone revision surgery or previous spinal fixation with an implant; being pregnant or hoping to become pregnant; or having a nickel, chromium, or Ti alloy allergy. Informed consent was obtained from each patient and, for patients younger than 18 years, informed consent was obtained from their parent or legal guardian.

The participants were allocated to two treatment arms: one group received $6.0-\mathrm{mm}$-diameter $\mathrm{CoCr}$ rods and the other received 6.0-mm-diameter Ti rods. At each institution, an independent administrator applied CliSSS Randoman software (Meditrix) to automatically randomize (1:1) participants stratified by age, height, weight, arm span, Lenke type, main Cobb angle, complications, lung capacity, and Risser grade. Participants remained blinded to their allocation throughout the study. Based on historical values, ${ }^{12} \mathrm{Ti}$ and $\mathrm{CoCr}$ rods were expected to produce $20^{\circ}$ and $30^{\circ}$ corrections in the main Cobb angles, respectively, with a standard deviation of $15^{\circ}$. For a desired power of 0.8 and an estimated $10 \%$ dropout rate, we estimated an optimal 40 cases to be required per cohort.

\section{Surgical Techniques}

All patients were treated at their institution of presentation, in a single stage by a specific senior spine surgeon at each affiliated institution following the predetermined trial procedures (UMIN Clinical Trials Registry UMIN000008838, registered on September 3, 2012). Under general anesthesia, each AIS patient was operated on via a standard posterior midline incision to reveal the thoracic vertebrae. Under neuromonitoring, uni- or polyaxial pedicle screws or hooks were applied according to the surgeon's discretion. For the 2 vertebrae above the thoracic apex on the concave side, caudally orientated uniplanar screws were required. Similarly, 2 uniplanar screws were applied below the apex. Direct vertebral rotation was allowed only near the apical vertebrae in the caudal direction. Accordingly, prebent 6.0-mm-diameter rods of either $\mathrm{CoCr}$ (Stryker) or Ti (Stryker) were used. Rods were secured into the anchor points, and direct vertebral rotation was applied using the SUK direct vertebral rotation spinal system (Stryker). A second rod composed of $\mathrm{CoCr}$ or $\mathrm{Ti}$ (matching the assigned cohort) was inserted to constrain the established correction. Fusion of the spine was performed according to the surgeon's expertise with either autografting inferior articular processes or Ponte osteotomy materials.

\section{Radiographic Analysis}

Patients were scheduled for follow-up assessments at their institution of treatment 2 weeks and 3, 6, and 12 months postsurgery. The primary outcomes were investigated using radiographic and CT images, which were obtained and analyzed by independent clinicians. Images 


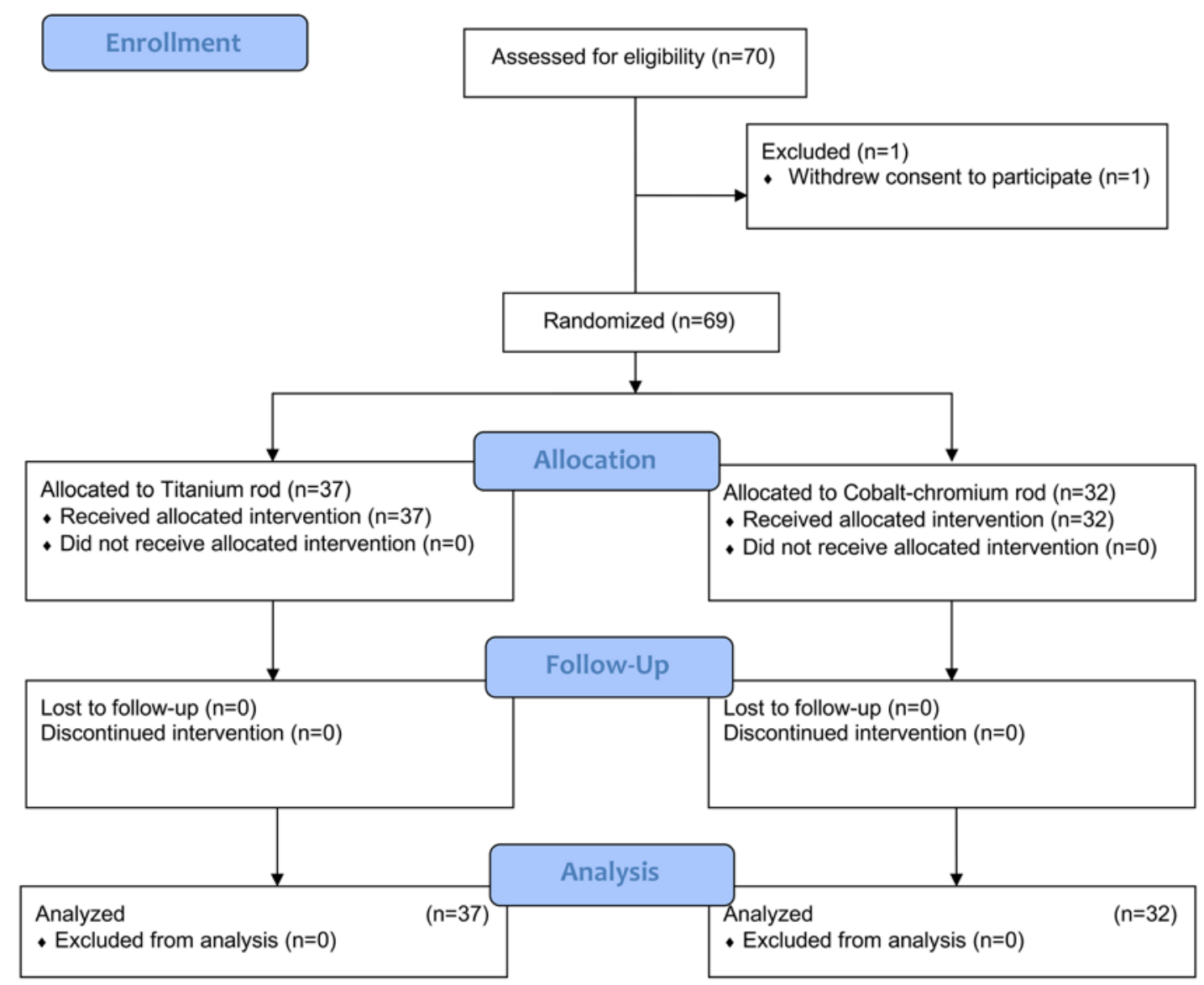

FIG. 1. Flowchart representation presenting the total number of participants enrolled and analyzed up to the final follow-up of the 1 -year study. The flowchart template is derived from the CONSORT 2010 guidelines for the transparent reporting of trials.

were used to determine the coronal main Cobb angle, sagittal thoracic kyphosis angle from T5 to T12, lumbar lordosis angle from $\mathrm{L} 1$ to $\mathrm{S} 1$, and apical vertebral rotation (AVR) following the method of Aaro and Dahlborn. ${ }^{13}$ Additionally, the rib hump size was determined, and the Cincinnati Correction Index was calculated as postoperative correction (\%)/preoperative flexibility (\%). ${ }^{14}$

\section{QOL Assessment}

As secondary outcomes, the patient's disability and general QOL were examined by applying questionnaires before surgery and at 6 and 12 months postsurgery. The Scoliosis Japanese Questionnaire-27, ${ }^{15}$ the Japanese version of the 12-Item Short-Form Health Survey (SF-12) ${ }^{16,17}$ and the Scoliosis Research Society-22r instrument (SRS-22r) ${ }^{18}$ were used for analysis of improvement in QOL parameters.

\section{Statistical Analysis}

The data were processed using Excel (Microsoft Corp.) and analyzed and visualized using Prism (GraphPad Software, Inc.). Baseline values were first compared between the two cohorts using the D'Agostino-Pearson test for normality. Parametric variables were compared using an unpaired t-test, and discrete or nonparametric variables were compared using the Mann-Whitney U-test. Temporal observations were analyzed using two-way ANOVA with Tukey's multiple comparison relative to the baseline test or Sidak's multiple comparison test to compare cohorts. All values are presented as the mean (SD) unless stated otherwise. Differences were considered to be significant at $\mathrm{p}$ $<0.05$.

\section{Results}

\section{Patient Recruitment}

In total, 70 patients were recruited, 69 of whom were eligible and included in the randomization (Fig. 1) to the $\mathrm{CoCr}(\mathrm{n}=32)$ or $\mathrm{Ti}(\mathrm{n}=37)$ cohort. Patients presented at a mean age of 14.5 years (2.3 years, range 10-20 years), body weight of $46.9 \mathrm{~kg}$ (7.1 kg, range 29-73 kg), and height of $155.2 \mathrm{~cm}(6.3 \mathrm{~cm}$, range $136.5-169.0 \mathrm{~cm})$. The respective Cobb angles were $56.9^{\circ}\left(9.5^{\circ}\right)$ and $58.5^{\circ}\left(2.4^{\circ}\right)$, for the $\mathrm{Ti}$ and $\mathrm{CoCr}$ cohorts, respectively. Spinal flexibility was also similar between cohorts: $39.6 \%(16.2 \%)$ and $37.7 \%$ $(16.3 \%)$, respectively $(\mathrm{p}=0.6369)$. None of the baseline variables differed significantly between the two cohorts (Table 1).

\section{Surgical Procedures}

All recruited patients received correction surgery ac- 
TABLE 1. Baseline demographics presented

\begin{tabular}{|c|c|c|c|c|c|c|c|}
\hline & \multicolumn{2}{|c|}{ Total Cohort } & \multicolumn{2}{|c|}{$\mathrm{Ti}$} & \multicolumn{3}{|c|}{$\mathrm{CoCr}$} \\
\hline & Mean (SD) & No. of Patients & Mean (SD) & No. of Patients & Mean (SD) & No. of Patients & $\mathrm{p}$ Value \\
\hline Age, yrs & $14.5(2.3)$ & 69 & $14.2(2.4)$ & 37 & $14.9(2.2)$ & 32 & $0.1627^{\star}$ \\
\hline Height, cm & $155.2(6.3)$ & 69 & $154.3(5.8)$ & 37 & $156.3(6.9)$ & 32 & $0.1777 \dagger$ \\
\hline Body weight, kg & $46.9(7.1)$ & 69 & $46.9(5.6)$ & 37 & $46.9(8.6)$ & 32 & $0.7444 \dagger$ \\
\hline BMI & $30.2(4.4)$ & 69 & $30.41(3.7)$ & 37 & $29.95(5.1)$ & 32 & $0.6748 \dagger$ \\
\hline Risser grade & $3.7(1.2)$ & 69 & $3.5(1.3)$ & 37 & $3.9(0.9)$ & 32 & $0.3177 \dagger$ \\
\hline Cobb angle, ${ }^{\circ}$ & $57.6(10.9)$ & 69 & $56.9(9.5)$ & 37 & $58.5(12.4)$ & 32 & $0.5360^{*}$ \\
\hline Active bending, ${ }^{\circ}$ & $35.8(13.4)$ & 69 & $34.7(12.1)$ & 37 & $37.1(14.9)$ & 32 & $0.4600^{*}$ \\
\hline Flexibility, $\%$ & $38.7(16.1)$ & 69 & $39.6(16.2)$ & 37 & $37.7(16.3)$ & 32 & $0.6369^{*}$ \\
\hline AVR, ${ }^{\circ}$ & $17.1(7.2)$ & 68 & $16.8(7.1)$ & 36 & $17.4(7.4)$ & 32 & $0.7439^{*}$ \\
\hline Cervical rotation, $^{\circ}$ & $5.5(3.7)$ & 63 & $5.2(3.5)$ & 33 & $5.9(4.0)$ & 30 & $0.5722 \dagger$ \\
\hline Thoracic kyphosis, ${ }^{\circ}$ & $16.7(13.6)$ & 68 & $16.0(11.7)$ & 37 & $17.6(15.6)$ & 31 & $0.9226 \dagger$ \\
\hline Rib hump, cm & $14.6(5.7)$ & 66 & $15.2(5.3)$ & 35 & $13.9(6.2)$ & 31 & $0.3723^{*}$ \\
\hline Arm span, cm & $155.0(8)$ & 57 & $154.7(8.3)$ & 32 & $155.4(8.3)$ & 25 & $0.7415^{*}$ \\
\hline Lung capacity, mL & $2407.1(416)$ & 68 & $2411(388)$ & 36 & $2403(452)$ & 32 & $0.9375^{*}$ \\
\hline Lenke classification & \multicolumn{6}{|c|}{ No. of Patients (\%) } & $p$ Value \\
\hline Type 1 & \multicolumn{2}{|c|}{$46(66.7)$} & \multicolumn{2}{|c|}{$24(64.9)$} & \multicolumn{2}{|c|}{$22(68.8)$} & \\
\hline Type 2 & \multicolumn{2}{|c|}{$20(29.0)$} & \multicolumn{2}{|c|}{$12(32.4)$} & \multicolumn{2}{|c|}{$8(25.0)$} & \\
\hline Type 3 & \multicolumn{2}{|c|}{$3(4.3)$} & \multicolumn{2}{|c|}{$1(2.7)$} & \multicolumn{2}{|c|}{$2(6.25)$} & $0.8809 \dagger$ \\
\hline Type 4 & \multicolumn{2}{|c|}{$0(0)$} & \multicolumn{2}{|c|}{$0(0)$} & \multicolumn{2}{|c|}{$0(0)$} & \\
\hline Type 5 & \multicolumn{2}{|c|}{$0(0)$} & \multicolumn{2}{|c|}{$0(0)$} & \multicolumn{2}{|c|}{$0(0)$} & \\
\hline
\end{tabular}

The $\mathrm{p}$ values indicate comparisons between the $\mathrm{Ti}$ and $\mathrm{CoCr}$ cohorts. No statistically significant differences were observed between the two experimental groups.

* Unpaired t-test for normal distributed values.

† Mann-Whitney test for nonparametric values.

cording to their allocated cohort (Fig. 2 and Table 2). Surgical procedures were similar between the two cohorts, with a mean blood loss of $765.3 \mathrm{~mL}(554.7 \mathrm{~mL}, \mathrm{p}=$ $0.6819)$ and a mean surgical time of 346.8 minutes $(87.3$ minutes, $p=0.6683$ ). The implant density (i.e., the number of hooks and screws per patient) was also similar ( $\mathrm{p}=$ 0.3853 ) with a mean density of 19.3 (3.7). A median of 11 (range 7-15) vertebral levels were fixed per patient, again showing no differences between the cohorts $(\mathrm{p}=0.4494)$. Four adverse events were recorded. In the Ti cohort, these included one serious adverse event of hemorrhagic shock, which was resolved by blood transfusion, and a superficial wound infection in another patient. In the CoCr cohort, the adverse events were once a superficial wound infection and once a deep wound infection. Wound infections were treated by wound debridement. All patients recovered and remained within their cohort, although 3 patients in the Ti cohort did not attend the final follow-up (Supplemental Table 1). Adverse events were not considered to be related to the experimental conditions.

\section{Radiographic Assessment}

The mean Cobb angles decreased significantly from the baseline to the 2-week follow-up: from $56.88^{\circ}\left(9.55^{\circ}\right)$ to $15.71^{\circ}\left(6.76^{\circ}\right)$ in the Ti cohort $(\mathrm{p}<0.001)$ and from $58.52^{\circ}$ $\left(12.36^{\circ}\right)$ to $16.92^{\circ}\left(7.94^{\circ}\right)$ in the CoCr cohort $(\mathrm{p}<0.001)$ (Fig. 3A and B). This significant decrease was maintained throughout and up to 12 months in the $\mathrm{CoCr}$ cohort, with mean Cobb angles of $18.15^{\circ}\left(6.03^{\circ}, \mathrm{p}<0.001\right)$ and $19.09^{\circ}$ $\left(7.18^{\circ}, \mathrm{p}<0.001\right)$, respectively. The mean thoracic kyphosis improved significantly at 2 weeks in the Ti cohort $\left(21.11^{\circ}\right.$ $\left[8.17^{\circ}\right], p=0.0057$ ), and this improvement was maintained throughout (Fig. 3C and D). Although the trend toward improvement was similar in the $\mathrm{CoCr}$ cohort, the results were not statistically significant, except for thoracic kyphosis at 6 months $\left(22.05^{\circ}\left[6.92^{\circ}\right], \mathrm{p}=0.0428\right)$. The lumbar lordosis decreased slightly at week 2 , but this decrease was not maintained throughout the follow-up (Fig. 3E and F). The rib hump decreased significantly in both cohorts (Fig. 3G and H). At 2 weeks, the mean AVR (Fig. 3I and J) decreased slightly in the Ti cohort, from $16.80^{\circ}\left(7.08^{\circ}\right)$ to $15.08^{\circ}\left(7.85^{\circ}\right)$, but the difference was not significant $(\mathrm{p}=$ 0.1996). In the CoCr cohort, the mean AVR decreased significantly from $17.37^{\circ}\left(7.36^{\circ}\right)$ to $14.55^{\circ}\left(7.43^{\circ}\right)(p=0.0143)$. The trend toward improvement was maintained for both cohorts up to 12 months. The overall mean correction rates at 12 months were $68.38 \%$ (9.93\%) and 67.06\% (11.66\%) for the $\mathrm{Ti}$ and $\mathrm{CoCr}$ cohorts, respectively (Fig. 3K). The Cincinnati Correction Index similarly did not result in any clear differences between the cohorts (Fig. 3L). For all radiographic parameters analyzed, no significant differences could be detected between the $\mathrm{Ti}$ and $\mathrm{CoCr}$ cohorts at any time point (Supplemental Table 1). A comparison of correction rates intrainstitutionally similarly showed no differences (Supplemental Fig. 1), except for a significant difference $(p=0.0225)$ in favor of the Ti cohort at 6 months 

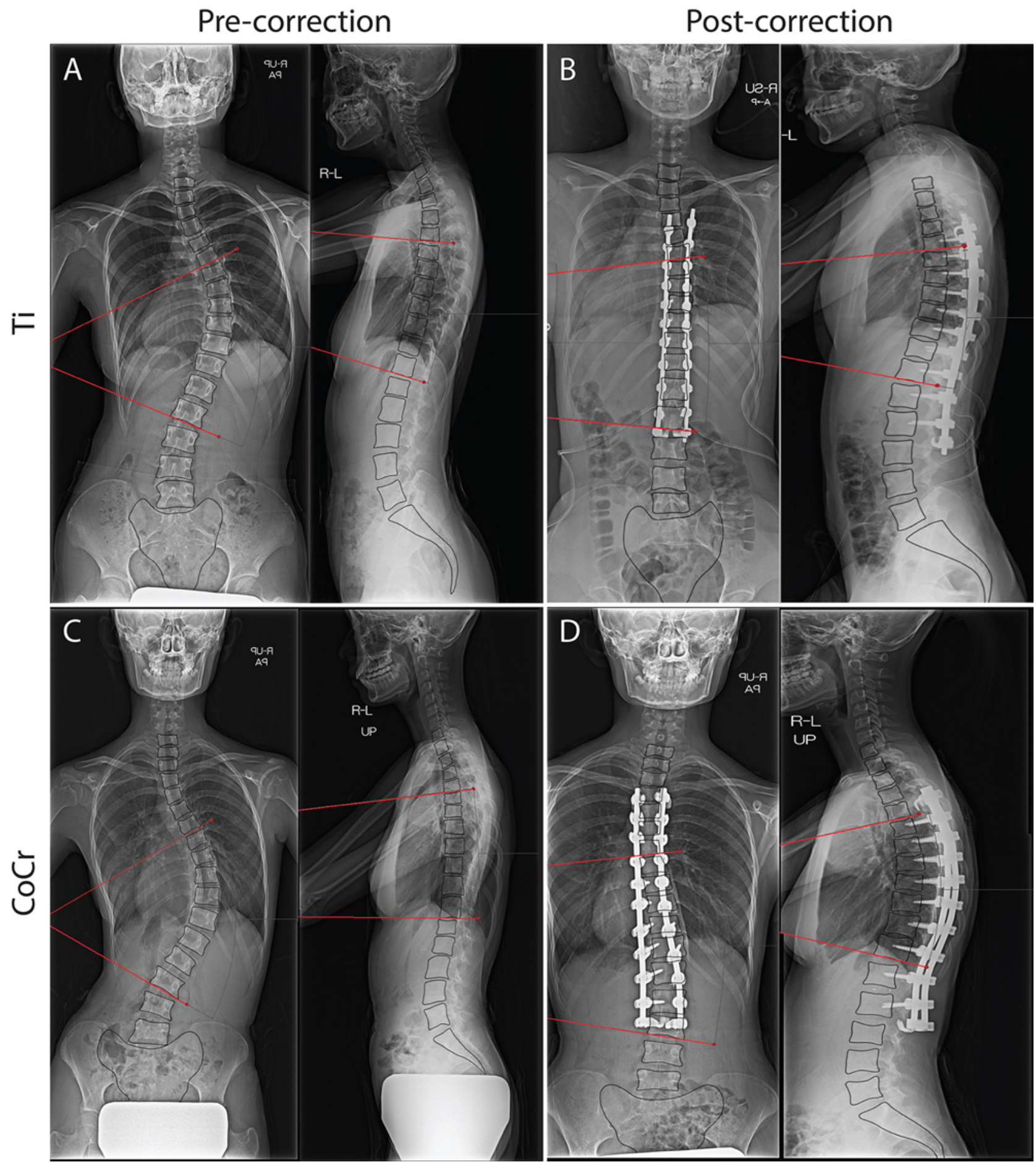

FIG. 2. Radiographs obtained in representative female patients with AIS who presented with abnormal curvature of the spine before the operation ( $A$ and $C$ ) and 1 year after the surgical correction (B and D). A: A female AIS patient in the Ti cohort presented with a thoracic $\mathrm{Cobb}$ angle of $47.55^{\circ}$ and thoracic kyphosis angle of $12.31^{\circ}$. B: Correction with Ti rods resulted in clear improvement in the coronal plane with a final Cobb angle of $11.75^{\circ}$ and thoracic kyphosis angle of $19.17^{\circ}$. C: A female AIS patient in the $\mathrm{CrCo}$ group presented with a Cobb angle of $57.20^{\circ}$ and thoracic kyphosis angle of $7.11^{\circ}$. D: Images obtained 1 year after the correction with $\mathrm{CoCr}$ rods, showing strong corrections, including a final thoracic $\mathrm{Cobb}$ angle of $16.64^{\circ}$ and thoracic kyphosis angle of $31.89^{\circ}$. Images were processed using Adobe Illustrator (Adobe Inc.). Red lines indicate the Cobb angle or thoracic kyphosis measurements on frontal and lateral plane images. 
TABLE 2. Characteristics of correctional surgery applied in both cohorts

\begin{tabular}{|c|c|c|c|c|c|c|c|}
\hline & \multicolumn{2}{|c|}{ Total Cohort } & \multicolumn{2}{|c|}{$\mathrm{Ti}$} & \multicolumn{3}{|c|}{$\mathrm{CoCr}$} \\
\hline & Mean (SD) & No. of Patients & Mean (SD) & No. of Patients & Mean (SD) & No. of Patients & p Value \\
\hline Blood loss, mL & $765.3(554.7)$ & 69 & 791.0 (581.8) & 37 & $735.6(529.4)$ & 32 & $0.6819^{*}$ \\
\hline Surgery time, mins & $346.8(87.3)$ & 62 & $351.6(81.2)$ & 31 & $342.0(94.0)$ & 31 & $0.6683^{*}$ \\
\hline \multirow[t]{2}{*}{ Implant density $†$} & $19.3(3.7)$ & 69 & $18.9(3.8)$ & 37 & $19.7(3.6)$ & 32 & $0.3853 \ddagger$ \\
\hline & Median [range] & No. of Patients & Median [range] & No. of Patients & Median [range] & No. of Patients & $p$ Value \\
\hline Deformity range§ & $8[5-10]$ & 69 & 8 [7-9] & 37 & $8[5-10]$ & 32 & $0.6518 \ddagger$ \\
\hline TK apex level & 9 [7-10] & 69 & 9 [7-10] & 37 & $8.5[7-10]$ & 32 & $0.1084 \ddagger$ \\
\hline \multirow[t]{2}{*}{ Fixed rangeף } & 11 [7-15] & 69 & $11[8-14]$ & 37 & 11 [7-15] & 32 & $0.4494 \ddagger$ \\
\hline & \multicolumn{6}{|c|}{ No. of Patients/Total No. of Patients (\%) } & $p$ Value \\
\hline Osteotomy & \multicolumn{2}{|c|}{$66 / 69(95.7)$} & \multicolumn{2}{|c|}{ 35/37 (94.6) } & \multicolumn{2}{|c|}{$31 / 32(96.9)$} & \\
\hline IAP & \multicolumn{2}{|c|}{$56 / 66(84.8)$} & \multicolumn{2}{|c|}{ 29/35 (82.9) } & \multicolumn{2}{|c|}{ 27/31 (87.1) } & $>0.999 \ddagger$ \\
\hline Ponte & \multicolumn{2}{|c|}{ 10/66 (15.2) } & \multicolumn{2}{|c|}{ 6/35 (17.1) } & \multicolumn{2}{|c|}{ 4/32 (12.5) } & \\
\hline
\end{tabular}

IAP = inferior articular process.

The $\mathrm{p}$ values indicate comparisons between the $\mathrm{Ti}$ and $\mathrm{CoCr}$ cohorts. No statistically significant differences were observed between the two experimental groups.

* Unpaired t-test for normal distributed values.

† The average implant density refers to each hook or pedicle screw applied per participant.

$\ddagger$ Mann-Whitney test for nonparametric values.

$\S$ The average number of vertebral levels involved in the scoliotic deformity.

IT The average number of vertebral levels fixed as part of the surgical correction.
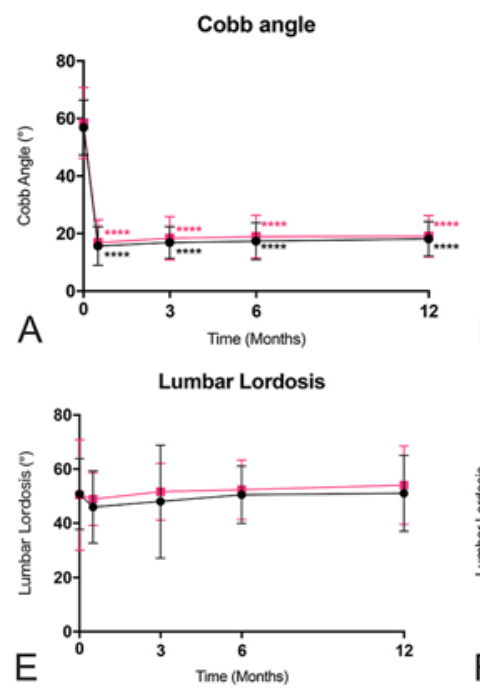

Apical Vertebral Rotation

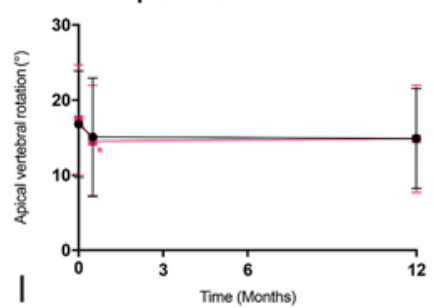

Mean difference Cobb Angle

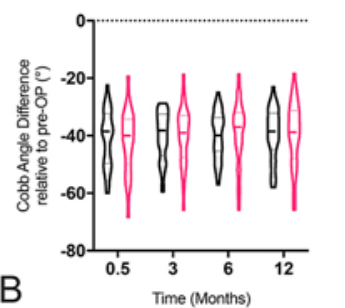

Mean difference Lumbar Lordosis

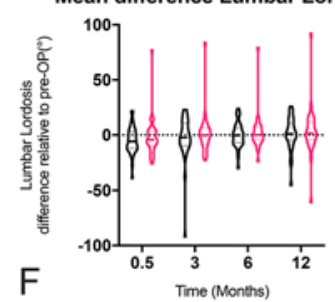

Mean difference Apical Vertebral Rotation

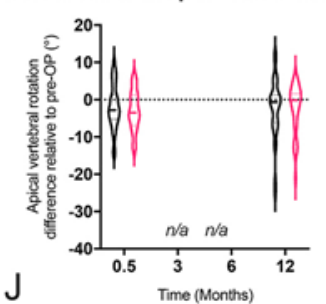

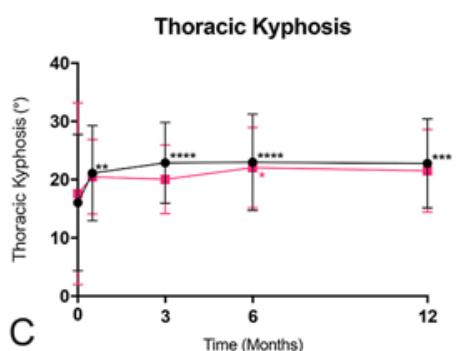

Rib Hump

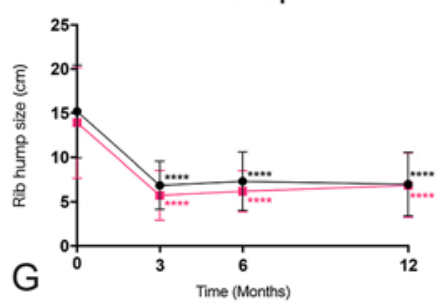

Correction rate

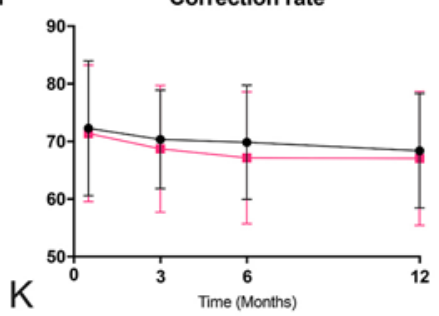

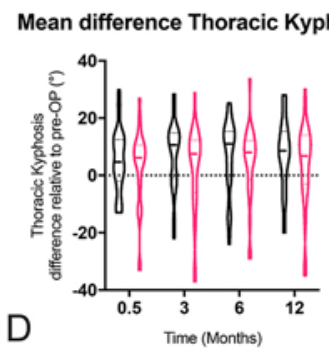

Mean difference Rib Hump

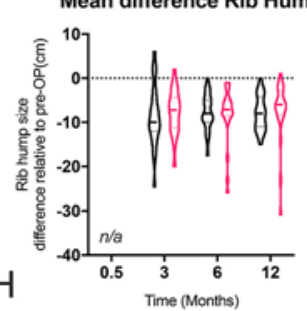

Cincinnati Correction Index

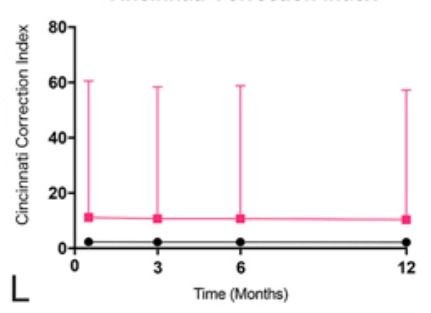

\section{$\mathrm{Ti}=\mathrm{CoCr}$}

FIG. 3. Graphic representation of changes in spinal deformities from the preoperative values to 2 weeks and 3,6 , and 12 months after the corrective surgery for the $\mathrm{Ti}$ (black) and $\mathrm{CoCr}(r e d)$ cohorts. Significance was calculated using two-way ANOVA with Tukey's multiple comparison relative to baseline values for each cohort. No significant differences were observed between cohorts. ${ }^{*} p<0.05 ;{ }^{* *} p<0.01 ;{ }^{* *} p<0.005 ;{ }^{* * *} p<0.001$. n/a $=$ not applicable. For a complete overview of the outcome values, see Supplemental Table 1. 
SF-12 Physical summary component

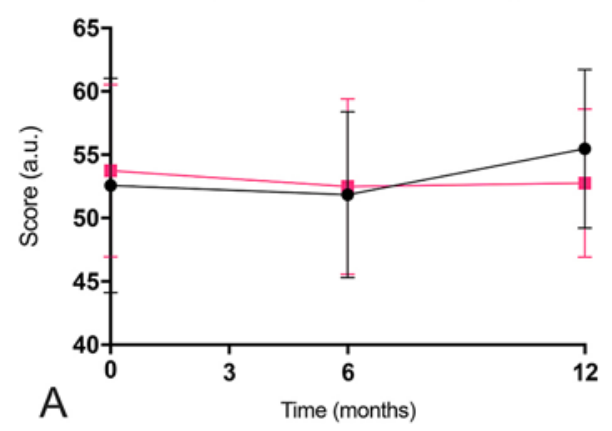

SRS-22r

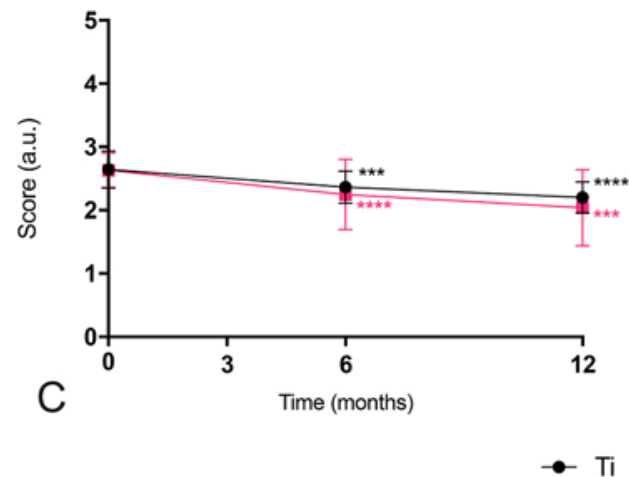

SF-12 Mental summary component

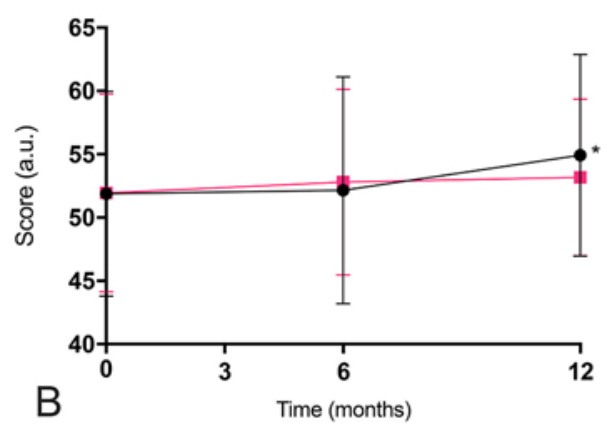

Scoliosis Japanese Questionnaire-27

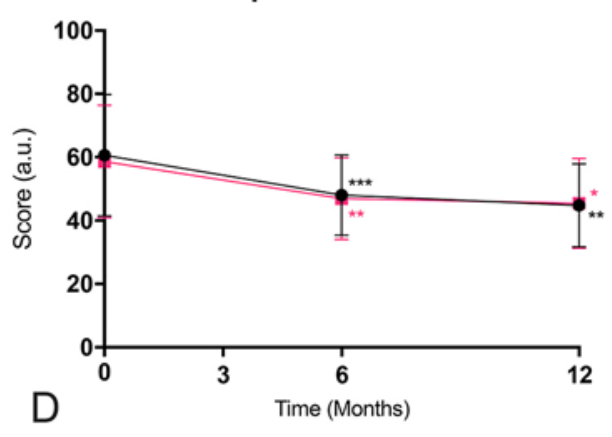

FIG. 4. Graphic representation of the changes in sum scores for the SF-12 physical component (A) and mental component (B) summaries, SRS-22r questionnaire (C), and Scoliosis Japanese Questionnaire-27 (D). The comparisons are between the preoperative scores and the 6 - to 12-month postoperative scores for the $\mathrm{Ti}$ (black) and $\mathrm{CoCr}$ (red) cohorts. Note that an increase in SF12 score indicates a reduction in negative symptoms, whereas a lower score on the SRS-22r and Scoliosis Japanese Questionnaire-27 indicates alleviation of symptoms. Significance was calculated using two-way ANOVA with Tukey's multiple comparison relative to baseline values for each cohort. No significant differences were observed between cohorts. ${ }^{*} p<0.05 ;{ }^{* *} p<0.01$; ${ }^{* *} p<$ $0.005 ;{ }^{* * *} p<0.001$. For a breakdown of scores, see Supplemental Figs. 2 and 3.

for 1 institution. At the 12-month follow-up, radiographic imaging revealed no apparent cases of nonfusion. No rod breakage or displacement was reported for either cohort.

\section{QOL Assessment}

The results of the SF-12 showed a trend toward a decline in the physical component in the $\mathrm{CoCr}$ cohort but a trend toward improvement in the Ti cohort (Fig. 4A). Both the $\mathrm{Ti}$ and $\mathrm{CoCr}$ cohorts showed a trend toward improvement in the SF-12 mental component summary, but this was significant at the final follow-up only in the Ti cohort (Fig. 4B). CoCr outcomes were significantly lower than $\mathrm{Ti}$ in subcomponents for pain and social life (Supplemental Fig. 2). Similarly, the results of both the SRS-22r and Scoliosis Japanese Questionnaire-27 declined significantly at 6 and 12 months after the operation, but these scores did not differ significantly between the two cohorts (Fig. 4C and $\mathrm{D}$ ). The sum scores decreased primarily because of decreases in the categories associated with mental health (Supplemental Figs. 2 and 3).

\section{Discussion}

Our findings show that both $\mathrm{Ti}$ and $\mathrm{CoCr}$ rods are suitable for engendering significant and clinically relevant anatomical corrections in AIS patients. We found no differences in the correction rates and QOL improvements between the two cohorts, suggesting that the stiffer $\mathrm{CoCr}$ rods have no clear additional benefits. Previously published retrospective studies have shown mixed results on this issue. Work by Angelliaume et al. in a cohort of 70 patients showed that $\mathrm{CoCr}$ rods did not improve coronal plane corrections but did modestly increase sagittal corrections. ${ }^{9}$ By contrast, a trial by Etemadifar et al. ${ }^{8}$ compared corrections by $\mathrm{CoCr}$ rods on the concave side ( $\mathrm{Ti}$ rods on the convex side) with those treated by only Ti rods and reported a small (but significant) improvement in the main curve correction by $\mathrm{CoCr}$, while Sabah et al. ${ }^{11}$ reported no differences in sagittal or coronal plane correction. Combined with our findings, it appears that there are no clear benefits in favor of the stiffer CoCr rods. Although counterintuitive, this is not surprising. Other studies were similarly unable to find that increased stiffness improved correction rates. For example, a large multicenter study by Prince et al. reported that neither coronal nor sagittal corrections were improved by increasing the rod diameter. ${ }^{19}$ A multicenter retrospective review of 275 AIS patients revealed no relationship of rod strength enhancing kyphosis corrections. ${ }^{20}$ These studies have suggested that the correction rates are mainly dependent on other factors, such 
as the preoperative flexibility, ${ }^{19}$ number of implants,,${ }^{19,21}$ or general surgical technique. ${ }^{20}$ This is further emphasized by our observation of differences in outcomes between institutions (Supplemental Fig. 1), further highlighting the need for multicenter clinical trials. Another possible reason for the lack of relationship between rod stiffness and spinal correction may be the limited rigidity of the spine. As the identified studies, including ours, involve young participants with a Lenke classification of 1-3, the strength of $\mathrm{Ti}$ implants may be sufficient to overcome the stiffness of the spine, and, thus, the additional stiffness of $\mathrm{CoCr}$ might not be of value. However, some have speculated that more rigid spines may benefit from the application of stiffer rods. Further study is needed to test this hypothesis.

Additionally, this the first study to examine QOL improvement differences resulting from $\mathrm{Ti}$ rod- and $\mathrm{CoCr}$ rod-mediated AIS corrections, which revealed no differences in outcomes (Supplemental Fig. 2). Although our trial is limited in size, the data do provide clear evidence that $\mathrm{Ti}$ and $\mathrm{CoCr}$ rods are similarly effective in engendering deformity corrections and improving QOL; thus, other material features take prominence. One consideration is the general higher cost of $\mathrm{CoCr}$. Also, $\mathrm{CoCr}$ has the disadvantage of exhibiting larger distortions on MRI compared with Ti rods, although the practical impact remains controversial. ${ }^{22}$ In addition, the use of $\mathrm{CoCr}$ rods appears to be associated with a higher prevalence of proximal junctional kyphosis (PJK). Two retrospective studies reported PJK rates of $46 \%$ in adults with spinal deformity ${ }^{23}$ and $60 \%$ for spinal fusion patients ${ }^{24}$ after CoCr rodinstrumented spine immobilization in contrast to rates of $18 \%$ and $27 \%$ following Ti rod corrections. However, both studies did link Ti rods to higher rates of rod breakage..$^{23,24}$ It should be noted, however, that rod breakage is likely of limited concern in AIS cases, as the involved spines are generally relatively flexible. For example, Ohrt-Nissen et al. noted that regarding rod fractures, it should "not be a main priority in choice of rod," as this is extremely rare in AIS patients. ${ }^{25}$ In addition, our study was unable to determine any difference in fusion rates, unsurprisingly, as pseudarthrosis rates are reported to be low in AIS deformity correction cases..$^{26,27}$ Finally, although the stiffer $\mathrm{CoCr}$ rods are expected to have enhanced profile maintenance and rod integrity after correction, previous reports do not support this notion. Work by Serhan et al. demonstrated that Ti rods are significantly better at retaining their shape than CoCr or SS rods and are less affected by rod flattening. ${ }^{28}$ Similarly, a case series by Sia et al. suggested greater deflection for $\mathrm{CoCr}$ rods. ${ }^{4}$ Moreover, $\mathrm{CoCr}$ rods are suggested to be more susceptible to loss of strength and stiffness as a result of rod contouring, which further challenges the switch toward $\mathrm{CoCr}$ over Ti rods. ${ }^{29}$ Considering the reported drawbacks of $\mathrm{CoCr}$ rods (e.g., MRI compatibility, cost, and rod deformity), the less stiff Ti rods might prove more beneficial for use in AIS corrective surgery.

Briefly, we would also like to comment on the prospective randomized controlled clinical trial nature of this study. Within the spine field, specifically for AIS, such trials are not very common. Moreover, their respective sample size tends to be small. This might in part be as- cribable to the relatively young age of AIS patients and general involvement of quite invasive (surgical) interventions, likely limiting participant recruitment. Our experience from this trial suggests that hesitation exists for the patients and their respective parent or legal guardian to participate. Nevertheless, for the trial presented here, we were able to attain a high recruitment rate $(69$ of 70 patients; Fig. 1). We believe that this is in part due to the design of our study, involving two "positive" treatment arms. Optimally, randomized controlled trials involve placebo, sham, or nontreatment controls; however, for recruitment of young patients as well as general ethical considerations, we believe that careful consideration should be given by clinicians and research on the types of treatments tested. Alternative strategies (e.g., crossover studies) might similarly be able to alleviate some of these hurdles. We strongly encourage other researchers, clinicians, and surgeons to evaluate new and established treatment strategies for AIS, to enhance our understanding on optimal strategies for resolving this complicated disorder and improve the quality of spine-related evidence-based medicine.

\section{Limitations}

Although our study comprises a prospective randomized multicenter clinical trial, and thus is able to alleviate most aspects of bias, some limitations should be considered when interpreting the results of our study. First, although our follow-up period of 12 months was sufficient to identify similar rates of correction and initial correction maintenance, a longer observation period could have given a better impression on the longevity of the corrections, in particular regarding the loss of rod shape, as this feature has been shown to result in enhanced outcomes for $\mathrm{Ti}$ rods compared with $\mathrm{CoCr}$ in previous studies. ${ }^{4,29}$ Second, our study is limited by its sample size, with 37 recruited patients in the Ti cohort and 32 participants in the $\mathrm{CoCr}$ group, even though it was estimated by our power calculation that 40 patients per cohort were needed. It should, however, be noted that these numbers are derived from calculations based on the assumptions of an estimated $10 \%$ dropout rate and a standard deviation in Cobb angle improvement of $15^{\circ}$. In our study, 66 of 69 patients reported at the final follow-up, which equals a dropout rate of $4.3 \%$. Moreover, Cobb angle standard deviations at the 12-month follow-up were on average $10.22^{\circ}$, which was lower than that used in the power calculation. Retrospectively applying these values for sample size calculation would severely reduce the estimated number of required participants, suggesting that the sample size used would be sufficient. Additionally, although the limited sample size could in part explain the lack of statistical significance when comparing outcomes between the two cohorts, regardless of statistical significance, the differences would still be clinically insignificant. For example, only a $0.08^{\circ}$ enhanced improvement in Cobb angle was observed in the Ti cohort compared with the CoCr rod-treated group (Supplemental Table 1), and such a difference likely would not have any clinical translation. Considering these two aspects, we believe our conclusion that Ti rod- and CoCr rod-mediated AIS corrections are equally effective is justified. Third, our study included three patients ( 1 in the Ti cohort and 2 in the 
$\mathrm{CoCr}$ cohort) who were classified as Lenke type 3. This classification generally involves stiffer spines with longer instrumentation and might therefore have increased the variability of the results. To determine if this was the case, both cohorts were also compared excluding these participants. This did not result in any evident clinical or statistical changes in outcomes (data not shown). Fourth, our study did not assess rates of PJK, a common complication in adult spinal surgery ${ }^{30}$ but also reported in AIS patients..$^{31,32}$ This is a particular shortcoming, as PJK in adult spinal surgery has been reported at higher frequencies with $\mathrm{CoCr}$ rods than with Ti rods and similarly stiffer instrumentation has been suggested as a risk factor. ${ }^{23,24}$ How the rod materials impact PJK rates following AIS surgery remains unknown and should be further examined.

In summary, although our study has some limitations, we do believe that by the nature of the trial design and extensive QOL and radiographic parameters analyzed, we were able to provide confident evidence that both $\mathrm{Ti}$ and $\mathrm{CoCr}$ rods are able to support similar spinal deformity corrections in AIS patients. For future work, we would recommend examining corrections over a longer period (> 2-year follow-up) between the Ti rod-and CoCr rod-treated groups, to analyze the effects of the different material on more rigid spines and larger deformity corrections, and to also evaluate rod shape retention and flattening during follow-up, to get a better grasp on the potential differences between $\mathrm{Ti}$ and $\mathrm{CoCr}$ rods for AIS corrections.

\section{Conclusions}

Our prospective randomized multicenter trial failed to demonstrate differences in improvements in QOL or spine deformity corrections between the $\mathrm{Ti}$ and $\mathrm{CoCr}$ cohorts. Our study combined with previous work provides clear evidence that both $\mathrm{Ti}$ and $\mathrm{CoCr}$ rod corrections can engender satisfactory clinical outcomes with no clear benefits of either rod for supporting sagittal and coronal plane corrections.

\section{References}

1. Konieczny MR, Senyurt H, Krauspe R. Epidemiology of adolescent idiopathic scoliosis. J Child Orthop. 2013;7(1):3-9.

2. Smit TH. Adolescent idiopathic scoliosis: the mechanobiology of differential growth. JOR Spine. 2020;3(4):e1115.

3. Lonner BS, Ren Y, Yaszay B, et al. Evolution of surgery for adolescent idiopathic scoliosis over 20 years: have outcomes improved? Spine (Phila Pa 1976). 2018;43(6):402-410.

4. Sia U, Tan BB, Teo YY, Wong CC. Post-implantation deformation of titanium rod and cobalt chrome rod in adolescent idiopathic scoliosis. Malays Orthop J. 2019;13(1):14-19.

5. Di Silvestre M, Bakaloudis G, Lolli F, Giacomini S. Latedeveloping infection following posterior fusion for adolescent idiopathic scoliosis. Eur Spine J. 2011;20(suppl 1):S121-S127.

6. Binyamin G, Shafi BM, Mery CM. Biomaterials: a primer for surgeons. Semin Pediatr Surg. 2006;15(4):276-283.

7. Warburton A, Girdler SJ, Mikhail CM, et al. Biomaterials in spinal implants: a review. Neurospine. 2020;17(1):101-110.

8. Etemadifar MR, Andalib A, Rahimian A, Nodushan SMHT. Cobalt chromium-titanium rods versus titanium-titanium rods for treatment of adolescent idiopathic scoliosis; which type of rod has better postoperative outcomes? Rev Assoc Med Bras (1992). 2018;64(12):1085-1090.
9. Angelliaume A, Ferrero E, Mazda K, et al. Titanium vs cobalt chromium: what is the best rod material to enhance adolescent idiopathic scoliosis correction with sublaminar bands? Eur Spine J. 2017;26(6):1732-1738.

10. Lamerain M, Bachy M, Delpont M, et al. CoCr rods provide better frontal correction of adolescent idiopathic scoliosis treated by all-pedicle screw fixation. Eur Spine J. 2014;23(6): $1190-1196$.

11. Sabah Y, Clément JL, Solla F, et al. Cobalt-chrome and titanium alloy rods provide similar coronal and sagittal correction in adolescent idiopathic scoliosis. Orthop Traumatol Surg Res. 2018;104(7):1073-1077.

12. Oto M, Shah SA, Mohammed MA, et al. Effectiveness and safety of posterior titanium instrumentation in children with adolescent idiopathic scoliosis: a prospective study. Eklem Hastalik Cerrahisi. 2011;22(3):124-128.

13. Aaro S, Dahlborn M. Estimation of vertebral rotation and the spinal and rib cage deformity in scoliosis by computer tomography. Spine (Phila Pa 1976). 1981;6(5):460-467.

14. Vora V, Crawford A, Babekhir N, et al. A pedicle screw construct gives an enhanced posterior correction of adolescent idiopathic scoliosis when compared with other constructs: myth or reality. Spine (Phila Pa 1976). 2007;32(17):1869-1874.

15. Doi T, Inoue H, Arai Y, et al. Reliability and validity of a novel quality of life questionnaire for female patients with adolescent idiopathic scoliosis: Scoliosis Japanese Questionnaire-27: a multicenter, cross-sectional study. BMC Musculoskelet Disord. 2018;19(1):99.

16. Ware JE, Kosinski M, Turner-Bowker DM, Gandek B. How to Score Version 2 of the SF-12 Health. QualityMetric Incorporated; 2000.

17. iHope International Corporation. SF-12 Health Survey. Accessed November 11, 2020. https://www.sf-36.jp/qol/sf12.html

18. Asher MA, Lai SM, Glattes RC, et al. Refinement of the SRS22 Health-Related Quality of Life questionnaire Function domain. Spine (Phila Pa 1976). 2006;31(5):593-597.

19. Prince DE, Matsumoto H, Chan CM, et al. The effect of rod diameter on correction of adolescent idiopathic scoliosis at two years follow-up. J Pediatr Orthop. 2014;34(1):22-28.

20. Monazzam S, Newton PO, Bastrom TP, Yaszay B. Multicenter comparison of the factors important in restoring thoracic kyphosis during posterior instrumentation for adolescent idiopathic scoliosis. Spine Deform. 2013;1(5):359-364.

21. Liu H, Li Z, Li S, et al. Main thoracic curve adolescent idiopathic scoliosis: association of higher rod stiffness and concave-side pedicle screw density with improvement in sagittal thoracic kyphosis restoration. J Neurosurg Spine. 2015;22(3): 259-266.

22. Ahmad FU, Sidani C, Fourzali R, Wang MY. Postoperative magnetic resonance imaging artifact with cobalt-chromium versus titanium spinal instrumentation: presented at the 2013 Joint Spine Section Meeting. J Neurosurg Spine. 2013;19(5): 629-636.

23. Han S, Hyun SJ, Kim KJ, et al. Comparative study between cobalt chrome and titanium alloy rods for multilevel spinal fusion: proximal junctional kyphosis more frequently occurred in patients having cobalt chrome rods. World Neurosurg. 2017;103:404-409.

24. Han S, Hyun SJ, Kim KJ, et al. Rod stiffness as a risk factor of proximal junctional kyphosis after adult spinal deformity surgery: comparative study between cobalt chrome multiplerod constructs and titanium alloy two-rod constructs. Spine J. 2017;17(7):962-968.

25. Ohrt-Nissen S, Dahl B, Gehrchen M. Choice of rods in surgical treatment of adolescent idiopathic scoliosis: what are the clinical implications of biomechanical properties? A review of the literature. Neurospine. 2018;15(2):123-130.

26. Lykissas MG, Jain VV, Nathan ST, et al. Mid- to long-term outcomes in adolescent idiopathic scoliosis after instrument- 
ed posterior spinal fusion: a meta-analysis. Spine (Phila Pa 1976). 2013;38(2):E113-E119.

27. Al-Mohrej OA, Aldakhil SS, Al-Rabiah MA, Al-Rabiah AM. Surgical treatment of adolescent idiopathic scoliosis: complications. Ann Med Surg (Lond). 2020;52:19-23.

28. Serhan H, Mhatre D, Newton P, et al. Would CoCr rods provide better correctional forces than stainless steel or titanium for rigid scoliosis curves? J Spinal Disord Tech. 2013;26(2): E70-E74.

29. Demura S, Murakami H, Hayashi H, et al. Influence of rod contouring on rod strength and stiffness in spine surgery. Orthopedics. 2015;38(6): $520-\mathrm{e} 523$.

30. Kim HJ, Iyer S. Proximal junctional kyphosis. J Am Acad Orthop Surg. 2016;24(5):318-326.

31. Kim YJ, Lenke LG, Bridwell KH, et al. Proximal junctional kyphosis in adolescent idiopathic scoliosis after 3 different types of posterior segmental spinal instrumentation and fusions: incidence and risk factor analysis of 410 cases. Spine (Phila Pa 1976). 2007;32(24):2731-2738.

32. Kim HJ, Lenke LG, Shaffrey CI, et al. Proximal junctional kyphosis as a distinct form of adjacent segment pathology after spinal deformity surgery: a systematic review. Spine (Phila Pa 1976). 2012;37(22)(suppl):S144-S164.

\section{Disclosures}

This study was funded in part by Stryker Corp. Drs. Tanaka, Takahashi, Taniguchi, and Takeshita received funding from Stryker Corp.

\section{Author Contributions}

Conception and design: Sakai, Tanaka, Takahashi, Taniguchi, Takeshita. Acquisition of data: Sakai, Tanaka, Takahashi, Taniguchi, Hiyama, Misawa, Kuraishi, Oba, Matsubayashi, Kato, Sugawara, Sato, Watanabe, Takeshita. Analysis and interpretation of data: Sakai, Schol. Drafting the article: Schol. Critically revising the article: all authors. Reviewed submitted version of manuscript: all authors. Approved the final version of the manuscript on behalf of all authors: Sakai. Statistical analysis: Schol. Administrative/technical/material support: Sakai, Tanaka, Takahashi, Taniguchi, Hiyama, Misawa, Kuraishi, Oba, Matsubayashi, Kato, Sugawara, Sato, Watanabe, Takeshita. Study supervision: Sakai, Tanaka, Takahashi, Taniguchi, Takeshita.

\section{Supplemental Information}

\section{Online-Only Content}

Supplemental material is available with the online version of the article.

Supplemental Table and Figures. https://thejns.org/doi/suppl/ 10.3171/2020.9.SPINE201486.

\section{Correspondence}

Daisuke Sakai: Tokai University School of Medicine, Kanagawa, Japan. daisakai@is.icc.u-tokai.ac.jp. 